\title{
Dynamic Simulation System Using DOF Picking Robot
}

\author{
Xiaojun Liu*
}

Department of Logistics and Information Management, Zhuhai College of Jilin University, Zhuhai 519041, Guangdong, China

\begin{abstract}
A visualized dynamic simulation platform of picking robot based on OpenGL and Matlab is established in order to verify the validity of kinematics analysis and visually reflect the motion process. The robot kinematic model is established with D-H method and the forward kinematic solution for the robot is obtained. The inverse kinematic solution is solved by using the simplified inverse transformation method according to the operation features of the robot. Solidworks is adopted to set up 3D robot model, which is transformed through Deep Exploration into cpp formatted files that can be recognized by OpenGL. Visualized simulation verification is conducted on the forward and the inverse kinematic solution and the grabbing motion of the manipulator are conducted on the simulation platform based on Visual $\mathrm{C}++6.0$ and OpenGL. Track planning is carried out for each of the manipulator joints by means of Matlab Robotics Toolbox. It is illustrated by the simulation results that the kinematic model established by D-H approach reflects the real motion conditions of the robot, and both the forward and inverse kinematic solutions are correct.
\end{abstract}

Keywords: DOF, Dynamic Simulation, OpenGL, Kinematic model, Matlab.

\section{INTRODUCTION}

As the largest vegetable producing and consuming country, China witnesses a continuous and stable development of vegetable production in recent years, with the cultivated area increasing from 95 million $m u$ in 1990 to 295 million $m u$ in 2011 and the yield from 195 million ton to 679 million ton. Meanwhile, however, it faces some challenges and problems demanding prompt solution, among which the most important issue is that the agricultural production technique is relatively backward and the level of mechanization and automation has a large gap with the developed countries. Agricultural producers endure high labor intensity; moreover, improper manual work methods damage the garden stuff vegetation and affect the quality and quantity of garden stuff production (X.Y. Tang, 2005) [1].

Harvesting or picking is the most effort-requiring and time-consuming task in vegetable production operation. Moreover, it requires picking in due time to guarantee the product quality, which is the most laborious in the whole operation. With the aging of population and the decrease of farming labor force, it is more and more significant to research and develop fruit and vegetable picking robot [2-5].

There appeared many picking robots at the end of last century at abroad. In particular, Japan is in the lead in this field in the world. The tomato picking robot developed by KONDO-N, et al. in Japan, is based on SCORBOT-ER industrial robot. (K.K. Guptal 2006) With 7 degree of

*Address correspondence to this author at the Department of Logistics and Information Management, Zhuhai College of Jilin University, Zhuhai 519041, Guangdong, China; Tel: 18163229635;

E-mail:1xj02041820@163.com freedom and equipped with 4 wheels, it can walk about in the field and conduct target location for picking through binocular vision. The eggplant picking robot, jointly developed by Japan National Institute of Vegetable and Tea and Gifu University, consist of ccd machine vision system, 5 DOF industrial manipulator, end effector and running gear, with a picking success rate of $62.5 \%$ and an operating speed of 64.1s/each.

In recent years, scholars in China also started the research and development of picking robot. Jian Song, etc., utilized optimization design method for the design of the robot body structure parameters in accordance with the eggplant growth and distribution space, and developed the basic machine of picking robot of 4 degrees of freedom. The overall testing system operates in stable and reliable way with a grabbing success rate of $89 \%$ and average elapsed time of $37.4 \mathrm{~s}$ (N. Kondo et al., 1996) [6, 7].

In the intelligent tomato picking robot end effector based on multi-sensor information fusion and open control system designed by Jizhan Liu, etc., its vacuum chuck device of the execution system can separate the fruit from the fruit bunch, its finger gripper mechanism can grasp the tomato firmly, and the fruit stem disconnecting device can cut off the fruit stem with laser (J.Z. liu et al. 2008) [8].

Vegetable picking robot works through the movement of each and every joint (Shigehiko et al. 2002). As an important constituent part of robotics, Robot kinematics mainly studies the relationships of the displacement, speed and acceleration of the connecting rods of the robot motion arm, whose purpose is to establish the relationships among spatial positions of the robot moving components and end effectors so as to 
provide theoretical basis and technical parameters for robot control (P. Cui 2011, Yang, Chifu 2010) [9].

In this paper, the author begins with establishing the robot mathematic model with Denavit-Hartenberg method, and applies OpenGL and Matlab to conduct kinematics analysis and simulation study on 5 DOF picking robot, which solves the problem of kinematics analysis such as tedious analysis procedure, large calculation amount and being Error-prone in traditional multi-rigid-body system. Meanwhile the kinematic performance of the robot movement mechanism is visually displayed in the form of simulation animation and diagram, providing a strong guarantee for the follow-up robot motion trail planning and the rationality verification of structure parameters [10].

\section{KINEMATICS ANALYSIS OF PICKING MANIPU- LATOR}

\subsection{Establishment of Robot Coordinate System}

The degree of freedom needed for the manipulator is analyzed and determined in accordance with the requirement of the eggplant picking process. The design requires for moderate amount of the degree of freedom without too many redundant ones so as to avoid the increase of control difficulty and structure complexity. This paper adopts five degree of freedom, namely, waist rotation, shoulder luffing, elbow luffing, wrist luffing and wrist turning, to match the grabbing process on the basis of actual demand. The structure is as shown in Fig. 1.

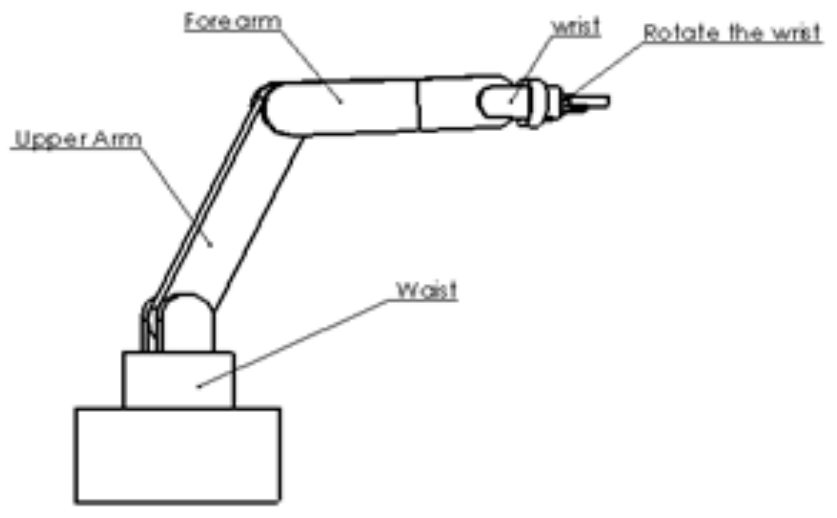

Fig. (1). Five degree of freedom picking manipulator schematic diagram.

The picking manipulator can be regarded as a connecting rod that is constituted with a series of joints. Each connecting rod establish a coordinate system. It adopts $4 \times 4$ homogeneous transformation matrix to describe the spatial position relationship of the adjacent robot rod pieces, thus translates the complicated kinematic question into equivalence transformation matrix of the reference coordinate system of the end effectors and reference coordinate system. Model of the connecting rod and joints is established according to Denavit-Hartenberg as shown in Fig. 2 [11].

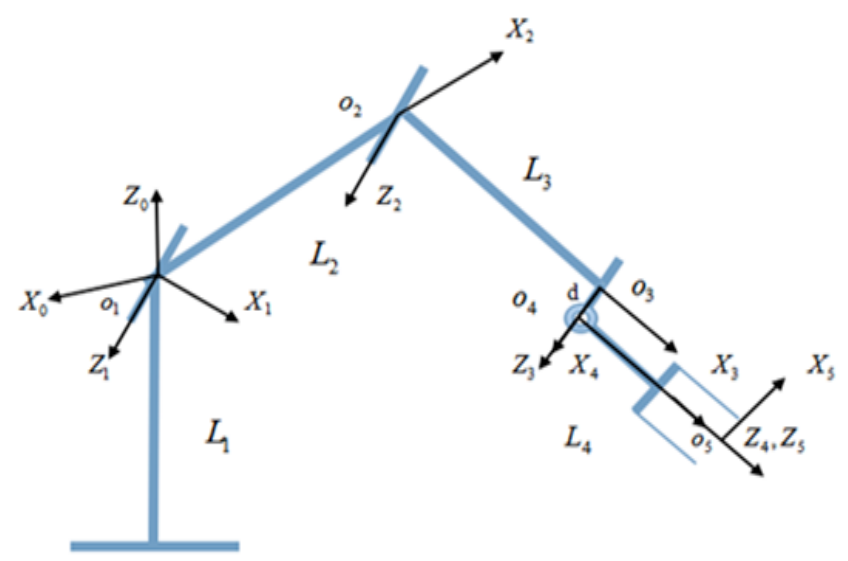

Fig. (2). Picking robot joint coordinate system.

From Fig. 1, according to D-H method define each connecting rod with 4 parameters: torsion angle $\alpha_{i}$, rod piece length $a_{i}$, joint angle $\theta_{i}$, horizontal distance $d_{i}$, all shown in Table 1.

\subsection{Kinematical Equation}

When the spatial relationship between the two adjacent connecting rods $i-1$ and $i$ changes in accordance with the following motions, the coordinate transformation can be accomplished.

1) Revolve $\theta_{i}$ around axes $Z_{\mathrm{i}-1}$ until it reaches the position where axes $X_{i-1}$ is parallel to axes $X_{i}$;

2) Translate distance $d_{i}$ along axes Zi-1 to cause Xi-1 to be collinear with $\mathrm{Xi}$;

3) Translate distance $\mathrm{a}_{\mathrm{i}}$ along Xi to cause the coordinate system origins of the connecting rods to be coincided;

4) Revolve angle $\alpha_{i}$ along $\mathrm{Xi}$ to cause axes $\mathrm{Zi}-1$ to be collinear with axes $\mathrm{Zi}$.

Repeat the above steps, and we can realize the transformation of coordinates from the robot base to the first joint, then to the second joint until the end effector. The 4 times of homogeneous transformation is presented in transformation matrix $\mathrm{A}_{\mathrm{i}}$

$$
\begin{aligned}
A_{i} & =\operatorname{Rot}\left(z, \theta_{i}\right) \times \operatorname{Trans}\left(0,0, d_{i}\right) \times \operatorname{Trans}\left(a_{i}, 0,0\right) \times \operatorname{Rot}\left(x, \alpha_{i}\right) \\
& =\left[\begin{array}{cccc}
\cos \theta_{i} & -\sin \theta_{i} \cos \alpha_{i} & \sin \theta_{i} \sin \alpha_{i} & a_{i} \cos \theta_{i} \\
\sin \theta_{i} & \cos \theta_{i} \cos \alpha_{i} & -\cos \theta_{i} \sin \alpha_{i} & a_{i} \sin \theta_{i} \\
0 & \sin \alpha_{i} & \cos \alpha & d_{i} \\
0 & 0 & 0 & 1
\end{array}\right]
\end{aligned}
$$

For the rotary joint, when the coordinate system of each robot connecting rod is specified, the parameters $\alpha_{i}, \alpha_{i}$ and $d_{i}$ are constant. In this case, matrix A becomes function of variable $\theta_{i}$. For the robot with $\mathrm{n}$ rotational joints, the general transformation of coordinates between the base and the end effectors is:

${ }^{0} T_{n}={ }^{0} T_{1}^{1} T_{2}^{2} T_{3} \cdot{ }^{n-1} T_{n}=A_{1} A_{2} A_{3} \cdots A_{n}$ 
Table 1. Picking robot joint parameter table.

\begin{tabular}{|c|c|c|c|c|c|c|}
\hline $\mathrm{i}$ & $\theta_{i}$ & $\alpha_{i}$ & $\alpha_{i}$ & $d_{i}$ & Variable Range & Link (mm) \\
\hline 1 & $\theta_{i}$ & $-90^{\circ}$ & 0 & 0 & $\pm 180^{\circ}$ & $\mathrm{L}_{1}=340 \mathrm{~mm}$ \\
\hline 2 & $\theta_{2}$ & 0 & 0 & 0 & $\pm 90^{\circ}$ & $\mathrm{L}_{2}=350 \mathrm{~mm}$ \\
\hline 3 & $\theta_{3}$ & 0 & $\mathrm{~L}_{3}$ & 0 & $\pm 150^{\circ}$ & $\mathrm{L}_{3}=350 \mathrm{~mm}$ \\
\hline 4 & $\theta_{4}$ & -90 & $\mathrm{~L}_{4}$ & $\mathrm{~d}$ & $\pm 120^{\circ}$ & $\mathrm{L}_{4}=0$ \\
\hline 5 & $\theta_{5}$ & 0 & 0 & 0 & $\mathrm{~L}_{5}=80 \mathrm{~mm}$ \\
\hline
\end{tabular}

According to equation (1) and equation (2), the picking robot with $5 \mathrm{DOF}$, and the end effector pose relative to the base coordinates can be expressed as (Xiong youping, 1996):

$$
\begin{aligned}
{ }^{0} T_{5} & =A_{1} A_{2} A_{3} A_{4} A_{5} \\
& =\left[\begin{array}{cccc}
c_{1} c_{234} c_{5}+s_{1} s_{5} & -c_{1} c_{234} s_{5}+s_{1} c_{5} & -c_{1} s_{234} & -d_{4} s_{1}+a_{3} c_{1} c_{23}+a_{2} c_{1} c_{2} \\
s_{1} c_{234} c_{5}-c_{1} s_{5} & -s_{1} c_{234} s_{5}-c_{1} c_{5} & -s_{1} s_{234} & d_{4} c_{1}+a_{3} s_{1} c_{23}+a_{2} s_{1} c_{2} \\
-s_{234} c_{5} & s_{234} s_{5} & -c_{234} & -a_{3} s_{23}-a_{2} s_{2} \\
0 & 0 & 0 & 1
\end{array}\right] \\
& =\left[\begin{array}{cccc}
n_{x} & o_{x} & a_{x} & p_{x} \\
n_{y} & o_{y} & a_{y} & p_{y} \\
n_{z} & o_{z} & a_{z} & p_{z} \\
0 & 0 & 0 & 1
\end{array}\right]
\end{aligned}
$$

The right-hand end of the equation is the end effector pose. The end effector pose matrix can be solved if the manipulator joint variables are known, and vice versa $(\mathrm{M} . \mathrm{Lu}$ 2011, Lara-Molina 2010).

The problem of the forward kinematic solution of the picking robot is to solve the pose of the end effector relative to the given coordinate system with the known joint variables and geometric parameters of the connecting rod. From formula 2 and formula 3 , the pose of the end effector of the 5 DOF picking robot is attained.

The issue of inverse robot kinematic solution is to determine the joint variable values with the given position and posture of the end effector relative to the spatial coordinate system with the given geometric parameters of the connecting rod of the robot. In this paper, a simplified inverse transformation method is adopted to solve the inverse kinematic solution of the robot. In this method, one or several inverse transformation matrixes are multiplied before the matrix transformation, and then the corresponding elements on both side of the equation are compared to achieve the goal of solving the inverse kinematic solution (W.Lu 2011).

\section{OPENGL SIMULATION SYSTEM}

OpenGL API provides some basic geometry shape drawing, for instance, dot, line, surface, cube, sphere, cylinder, circular cone, etc. Nevertheless, it will be a difficult and tedious process to only use the built-in API for modeling when constructing complicated robot geometry shapes. In this article solid works is selected for modeling, which then trans- forms the component format through Deep Exploration into cpp formatted files that can be recognized by OpenGL, displays and assembles the model at appropriate position in MFC through calling glCallList(), and imports the model into OpenGL, as shown in the flow chart (Fig. 3).

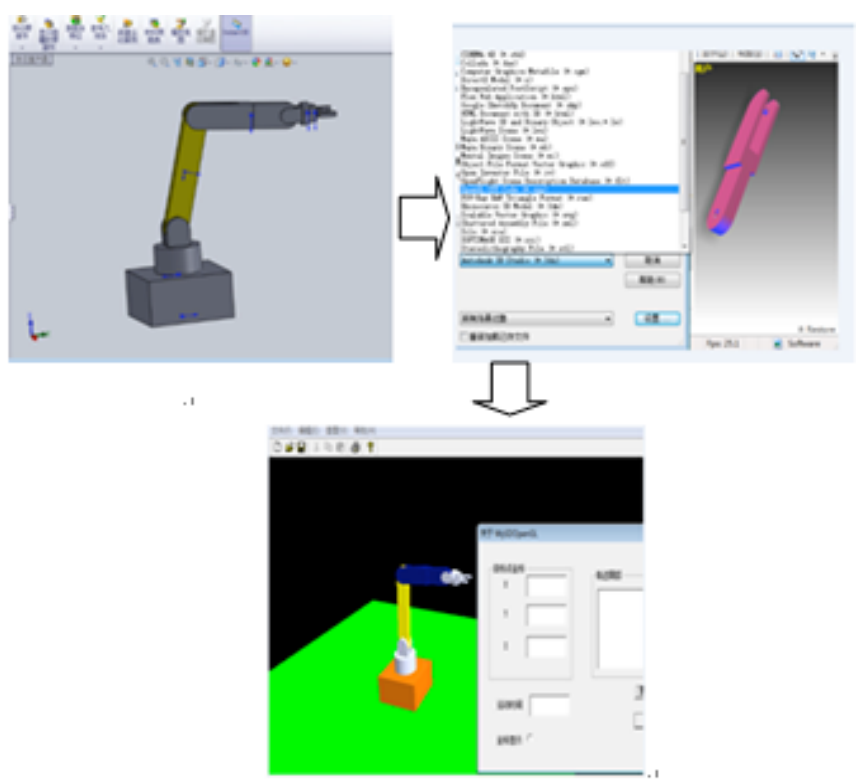

Fig. (3). The flow chart of model import OpenGL.

\subsection{Implementation of Dynamic Simulation}

OpenGL software package adopted in this article is independent of hardware and can operate in several operating systems, so it does not have interface problems. The steps of combing OpenGL and windowsGDI are as follows.

1) Call function setpixelFomart and set pixel format;

2) Establish drawing description list;

3) Acquire equipment list and correlate it to description list;

4) Call OpenGL command to conduct drawing job;

5) Call swapBuffer to refresh contents on the screen;

6) Release DC and RC when finishing;

As the positive direction of $\mathrm{Z}$ axis of 3-D coordinate system in OpenGL faces the screen and the observer, it is not 
corresponding to the coordinate system used by the manipulator. Use translation and rotation function to transform anew the coordinate, as shown in Fig. 4.
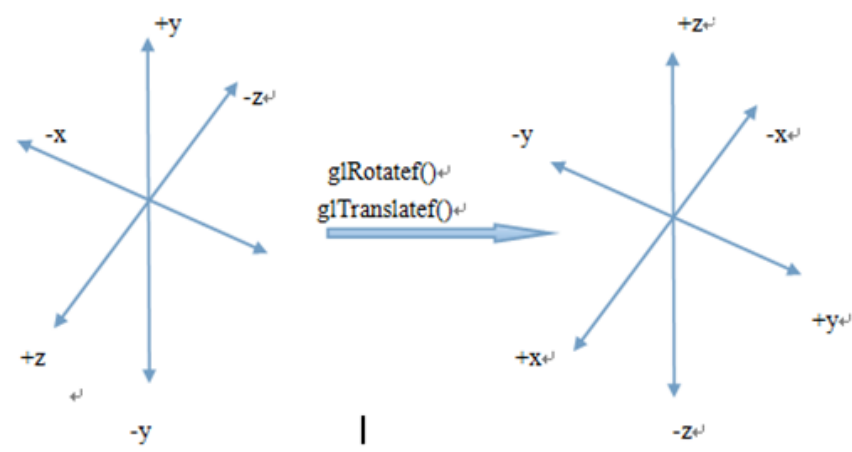

Fig. (4). Waist coordinate system transformation.

For the sake of the troubles for drawing that every time call the rotation and translation functions, they will be accumulated to the model view matrix in OpenGL. OpenGL supplies a unit matrix to reset the origin, i.e., resetting the coordinate system origin by multiplying with the unit matrix. Load unit matrix code is as follows:

- glMatrixMode();

- $\quad$ glLoadIdentity();

In order to conveniently load unit matrix into the matrix stack, glPushMatrix () is employed to press the present matrix into the matrix stack. Call glPushMatrix () is used to revert the matrix to the original state when you want to recover the present matrix.

For displaying animation effect, OpenGL provides double buffers---when it displays foreground buffering one image, the background buffer is drawing the next image, --which enables the animation to be consistent. Define a timer and call SwapBuffer()。

\subsection{Simulation Result and Analysis}

Acquire the robot end effector position and pose relative to reference coordinate system, i.e., the pose matrix, with given variables of the joints linking the connecting rods in the simulation system. Meanwhile, in accordance with the five given joint angles, each robot joint rotates correspondingly in the simulation platform so as to get a new position and pose.

\subsubsection{Kinematics Inverse Solution and Grabbing Motion Verification}

According to the requirements of picking object identification and location result, seek the robot kinematics inverse solution using the kinematics inverse solution method proposed in this article. Transfer respectively inverse solution result to the picking robot. Define the coordinates of the target grabbing point in procedure. The computer acquires the inverse solution and track planning, conducts dynamic simulation, completes the target grabbing and verifies kinematics inverse solution algorithm. Fig. (5) shows the animation simulation of the manipulator fetching process. It can be seen from the OpenGL visualized simulation result that the inverse solution method proposed in this article is simple and effective with accurate result.

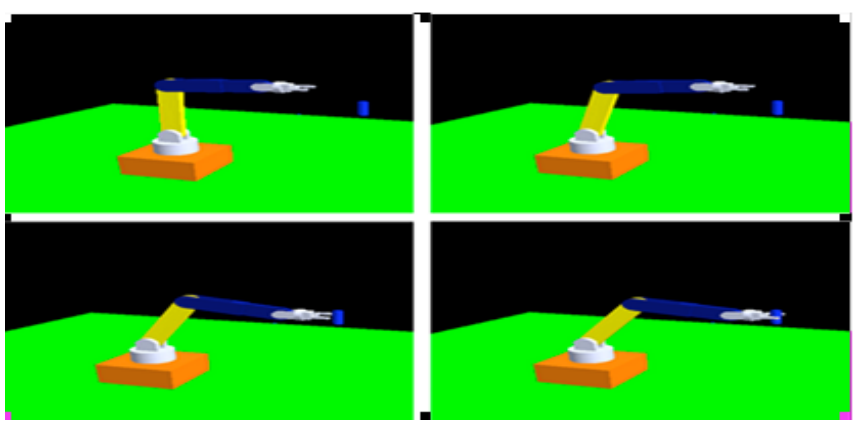

Fig. (5). Animation simulation manipulator fetching process.

\section{MANIPULATOR KINEMATICS PARAMETER VERIFICATION AND SIMULATION}

The robot kinematics forward and inverse solutions are verified through OpenGL-based robot dynamic simulation. However, it does not obtain the relation curve of the picking robot displacement, speed and accelerated speed. Matlab Robotics Toolbox developed and maintained by Australian scientist Peter Corke is a series of Matlab-based robotics toolbox, which is convenient for robot modeling and getting kinematic parameter curves of each joint (Corke et al. 1995).

\subsection{Robotics Toolbox Modeling}

When using Robotics Toolbox to structure a robot object, it firstly needs to build each joint and then implements the whole robot object by means of joint combination. To completely structure a robot object, it needs D-H parameters, joint types (translation or revolution joint), joint quality and gear ratio [12].

Robotics Toolbox modeling is proceeded according to D-H parameter list. Set command as follows:

\section{$\mathrm{L}=\mathrm{LINK}([$ alpha A theta D sigma], CONVENTION);}

Among which, alpha A theta D sigma represent respectively torsion angle, rod piece length, joint angle, horizontal distance, joint type; CONVENTION represents D-H type.

The Matlab-based picking robot model established as shown in Fig. 6 is composed of the most basic elements of joint-type robot- joints and connecting rods. It is in original state, i.e. all joint moves and angles are zero.

\subsection{Track Planning and Simulation}

Picking robot motion tracks fall into two types --- space joint track and Cartesian space track. Robot track planning is to work out the position, speed and accelerated speed needed by the joints in motion in order to complete the operation. Joint space track planning is chosen because it is too complex for planning in Cartesian space and liable to have cases of no solution. 


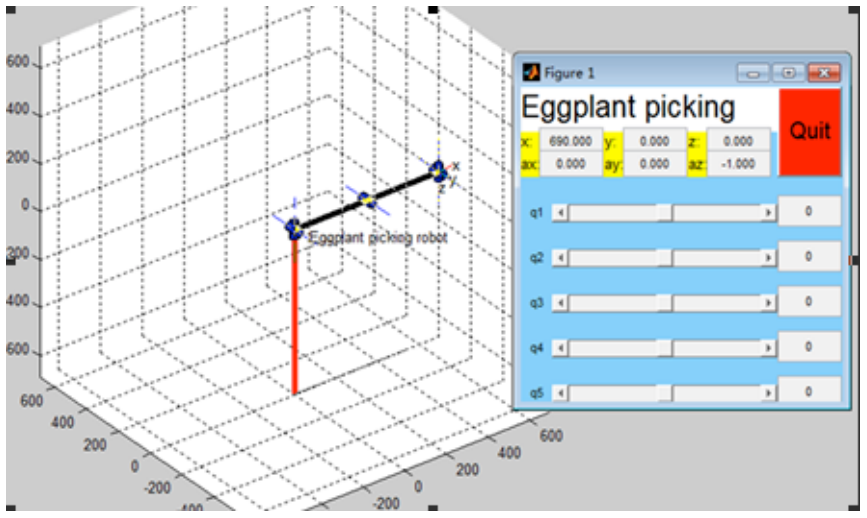

Fig. (6). Manipulator mod.

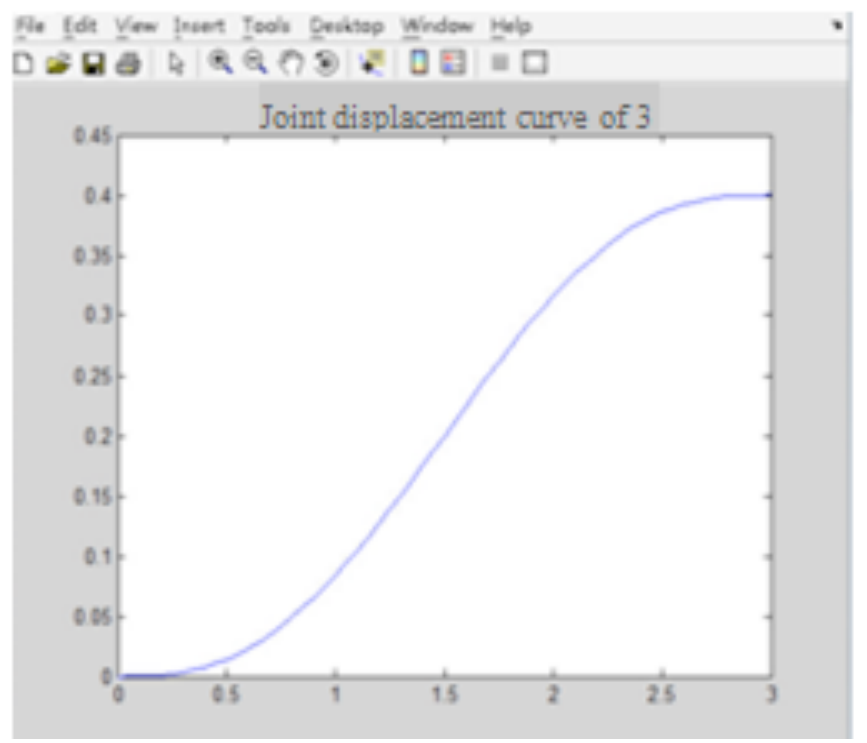

Fig. (7). Joint displacement curve of 3.

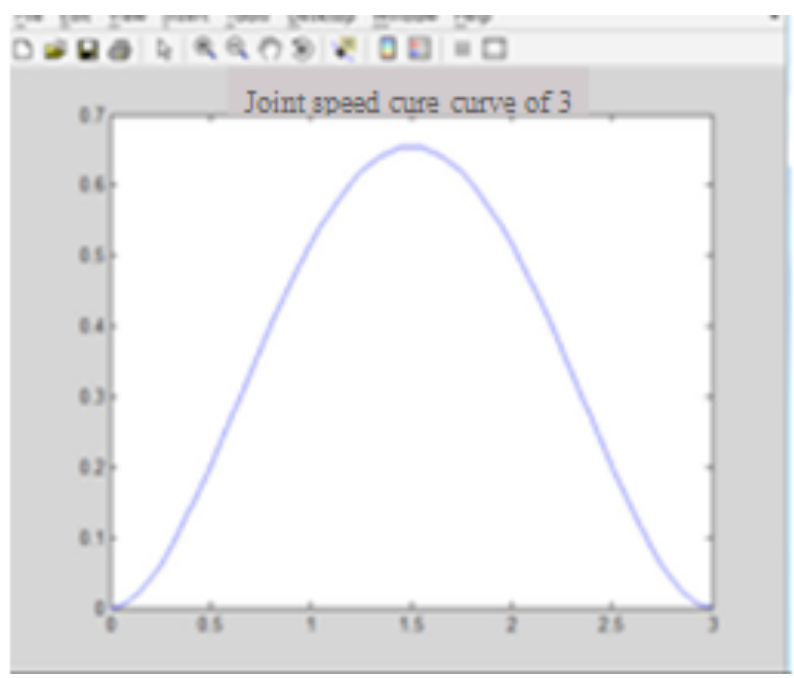

Fig. (8). Joint speed cure curve of 3 .

The toolbox provides function jtraj for interpolation to the joint, whose call format is $[\mathrm{q}, \mathrm{qd}, \mathrm{qdd}]=\mathrm{jtraj}(\mathrm{qr}, \mathrm{qz}, \mathrm{t})$;

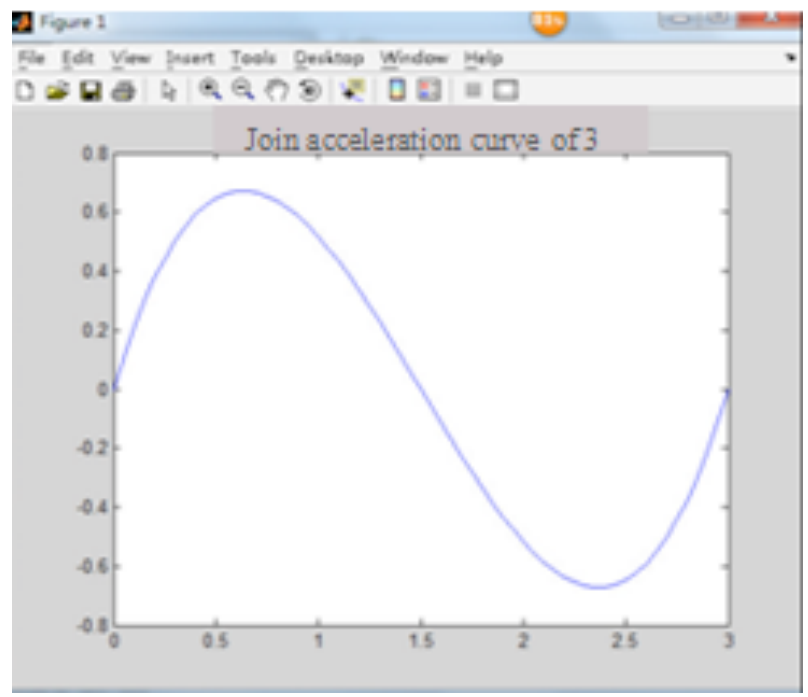

Fig. (9). Join speed t curve of 3.

Q matrix row represents the rotation angle of each joint of the actual sampling point, and the latter two represent respectively speed vector and accelerated speed vector.

The tracks of Joint 3 in sampling time from the initial position qr to the final position qz are as shown in Figs. 7-9. It is observed that the end effector does not produce strenuous vibration during the entire motion procedure meeting the job requirement.

It is verified that the theoretical calculation results obtained from (4), (5) in D-H coordinate system is identical with the simulation result.

Displacement and speed curve chart can reflect the real motion conditions and be applied to picking robot control.

\section{CONCLUSION}

Picking robot of $5 \mathrm{DOF}$ is developed in order to improve the economy and adaptability of the picking robot. It adopts 5-DOF open motion chain which connects the waist, the big arm, the small arm and the wrist in series through the rotational joints. The robot completes the picking operation through movements of each joint. The objective of studying robot kinematics is to establish the relationship of the spatial position between the robot motion components and the end effector, to build mathematic model of the robot arm movements, and provide theoretical basis and technical parameters for robotic control.

It applies dynamic simulation technology based on OpenGL and Matlab to the design and develop picking robot, displays dynamically the whole mechanism motion process, verifies the validity of the control algorithmic, and obtains the robot kinematic parameter curve. It is illustrated by the simulation results that the kinematic model established by $\mathrm{D}-\mathrm{H}$ approach reflects the real motion conditions of the robot, and both the forward and inverse kinematic solutions are correct. The research applies computer dynamic simulation technology to agricultural robot design. Compared with the traditional method of manufacturing trial product, it not only saves the input of manpower and material resources, but 
also greatly shortens the mechanism design and development cycle. As a modernized design means, virtual design technology will certainly have an important influence on the development of agricultural equipment.

\section{CONFLICT OF INTEREST}

The author confirms that this article content has no conflict of interest.

\section{ACKNOWLEDGEMENTS}

This work is supported by Shandong province science and technology development project (No.2011YD03048) and Shandong Provincial Natural Science Foundation, china (No.Y2008G32) and Shandong Provincial universities Scientific Research Project (No.J09LG53).

\section{REFERENCES}

[1] P. Corke, "A computer tool for simulation and analysis: the Robotics Toolbox for MATLAB," In: Proceedings of the National Conference on Australian Robot Association, 1995.

[2] J. Z. Liu, P. P. Li, and Z. G. Li, "Hardware design of the endeffector for tomato-harvesting robot," Transactions of the Chinese Society for Agricultural Machinery, vol. 39, no. 3, pp. 109-112, 2008.
[3] K. K. Gupta, and R. Gupta, "Wavelet based speckle filtering of the SAR images," International Review on Computers and Software, vol. 1, no. 3, pp. 224-232, 2006.

[4] F. A. Lara-Molina, J. M. Rosário, and D. Dumur, "Architecture of predictive control for a Stewart platform manipulator," $8^{\text {th }}$ World Congress on IEEE Intelligent Control and Automation (WCICA), 2010, 2010.

[5] M. Lu, and H.J. Zhao, "Modified self-adaptable wavelet transform arithmetic in image edge detection," Application Research of Computers, vol. 28, no. 1, pp. 385-387, 2011.

[6] N. Kondo, M. Monta, T. Fujiura, "Fruit harvesting robot in Japan," Advances in Space Research, vol. 18, no. 2, pp. 181-184, 1996.

[7] P. Cui, Z. Chen, and X.C. Zhang, "Statics analysis of apple-picking robot humanoid manipulator," Transactions of the Chinese Society for Agricultural Machinery, vol. 42, no. 2, pp. 149-153, 2011.

[8] S. Hayashi, K. Ganno, and Y. Ishii, "Robotic harvesting system for eggplants," JARQ, vol. 36, no. 6, pp. 163-168, 2002.

[9] X.Y. Tang, and T.Z. Zhang, "Robotics for fruit and vegetable harvesting: a review," Robot, vol. 27, no. 1, pp. 90-96, 2005.

[10] Y. Xiong, "Roboics" Chain Machi Press, China, pp. 145-155.

[11] Y. Chifu, Y. Zhengmae, O.O. Peter, and H. Junwei, "Modeling and simulation of spatial 6-DOF parallel robots using Simulink and Simmechanics," In: $3^{\text {rd }}$ IEEE International Conference on Computer Science and Information Technology (ICCSIT), vol. 4, pp. 444448, 2010.

[12] W. Lu, A.G. Song, and J.R. Cai, "Structural design and kinematics algorithm research for orange harvesting robot," Journal Of Southeast University, vol. 41, no. 1, pp. 95-100, 2011.

(C) Xiaojun Liu; Licensee Bentham Open.

This is an open access article licensed under the terms of the (https://creativecommons.org/licenses/by/4.0/legalcode), which permits unrestricted, non-commercial use, distribution and reproduction in any medium, provided the work is properly cited. 